\title{
Activity of Daily Living and Motor Evoked Potentials in the Subacute Stroke Patients
}

\author{
Kil Byung Lim, MD, Jeong-Ah Kim, MD
}

\author{
Department of Rehabilitation Medicine, Inje University Ilsan Paik Hospital, \\ Inje University College of Medicine, Goyang, Korea
}

Objective To investigate the effectiveness of the daily living activity and motor evoked potential (MEP) in the subacute stroke patients.

Methods Nineteen subjects with subacute ischaemic/hemorrhagic stroke developed in the last three months were enrolled, and MEP was measured with transcranial magnetic stimulation. Functional Independence Measure (FIM) score were evaluated in both groups before and 4 weeks after comprehensive rehabilitative management. According to the presence of MEP response in the affected hemisphere, subjects were divided into MEP positive and negative group.

Results There was no significant difference between the two groups in age, sex, and post-onset duration. Four weeks later, the change in total FIM and self-care score improved significantly in the MEP-positive group, when compared to the MEP-negative group $(\mathrm{p}<0.05)$. However, cognitive improvement had no relationship with MEP responsiveness.

Conclusion We concluded that initial measurement of MEP is a useful assessment tool in predicting functional outcome of subacute stroke patients.

Keywords Stroke, Hemiplegia, Motor evoked potentials, Activities of daily living

\section{INTRODUCTION}

The stroke is a disease caused by a cardiovascular accident. It is a clinical symptom in which a sudden

Received June 22, 2012; Accepted September 6, 2012

Corresponding author: Jeong-Ah Kim

Department of Rehabilitation Medicine, Inje University Ilsan Paik Hospital, Inje University College of Medicine, 170 Juhwa-ro, Ilsanseo-gu, Goyang 411-706, Korea

Tel: +82-31-910-7440, Fax: +82-31-910-7746, E-mail: wholespirit@ hanmail.net

(c) This is an open-access article distributed under the terms of the Creative Commons Attribution Non-Commercial License (http://creativecommons. org/licenses/by-nc/3.0) which permits unrestricted noncommercial use, distribution, and reproduction in any medium, provided the original work is properly cited.

Copyright $\odot 2013$ by Korean Academy of Rehabilitation Medicine neurological impairment takes place, and gives rise to decreased consciousness, cognitive impairment, movement disorder, and speech disorder. It will lead to gait disturbance and disability in activities of daily living, and deteriorate the quality of life of the stroke patient. The accompanying cognitive impairment will make it difficult for the patient to return to society, and affect the will to go through rehabilitation and the acquisition of motor skills [1]. Accordingly, predicting the recovery of motor skills and cognitive functions after the brain damage in a hemiplegic patient who has had a stroke is very important to establishing the treatment goal and plan for the patient.

The Glasgow Coma Scale, age, degree and duration of coma, brainstem reflexes, and motor responses are used 
as prognostic factors for the recovery of functions after brain damage [2]. As these factors cannot be correctly tested when a patient is in a coma or a tranquilizer is administered, the brain computed tomography (CT), brain magnetic resonance imaging (MRI), and evoked potential have been used as more objective tests. Among these, the motor evoked potential is obtained by using transcranial magnetic stimulation to excite the cerebral motor cortex, and the presynaptic motor neuron of the cerebral cortex is stimulated to measure the latent time and amplitude. It is used to diagnose myelopathy, brain lesion, and peripheral neuropathy, and judges the degree of functional recovery, predicting the prognosis in the early phases of the stroke [3,4]. Previous studies on the motor evoked potential reported that the patients with reactions in the motor evoked potential of the upper limb and the lower limb had better prognosis for functional recovery than those with no reaction $[5,6]$. Other studies reported that it is more beneficial to conduct both the somatosensory evoked potential and the motor evoked potential at the same time to more accurately predict the recovery of the motor skills of the plegic upper $[7,8]$.

The indicators for evaluating the patient's functional recovery are the Functional Independence Measure (FIM) score, and the Modified Barthel Index (MBI). The FIM scale includes the physical, cognitive, linguistic, and social aspects, and MBI includes only the physical aspects $[9,10]$. Previous studies used MBI to predict prognosis according to the motor evoked potential, but there was no study that compared the FIM score and the degree of improvement in detailed FIM subitems. Therefore, we tried to understand the recovery of the motor skills, cognitive, linguistic, and social aspects by comparing the FIM and the subitems of FIM in the hemiplegic patients who had a stroke, according to the presence or absence of the motor evoked potential.

\section{MATERIALS AND METHODS}

The subjects were patients who were first diagnosed with cerebral hemorrhage or cerebral infarction in the middle cerebral artery and basal ganglia, subsequently hospitalized and received comprehensive rehabilitation between May 2008 and May 2010. Patients who had convulsion, diabetes or peripheral neuropathy, patients who were delirious or could not sit stably for more than
30 minutes, and patients who had a history of orthopedic surgery were excluded from the study.

All patients had the motor evoked potential test between 1 and 2 months after the occurrence of their disease, which corresponded to the subacute period. The magnetic stimulus during the motor evoked potential used Magstim 200 (Magstim, Dyfed, UK) and a coil stimulator with a radius of $9 \mathrm{~cm}$, and an electromyogram (Medelec Synergy, Oxford, UK) was used to record the motor evoked potential. The active electrode is placed in the center of the abductor pollicis brevis of the plegic hand, and the reference electrode is placed at its distal end, so that the electric potential recorded at the muscle may be recorded. As for the magnetic stimulus, the authors placed the center of the coil in the parietal region of the head opposite the paralyzed upper limb. While moving it at intervals of $1 \mathrm{~cm}$ and increasing the $50 \%$ stimulus by $2 \%$ at a time, the authors repeated the stimulus until they reached the maximal stimulus of $100 \%$; and the minimal stimulus with the evoked stimulus, recorded at the abductor pollicis brevis, observed at above $50 \mu \mathrm{V}$ more than 5 out of 10 times, became the resting threshold. This location was selected as the stimulus site, and the mean of the interspinous amplitude of the motor evoked potential, obtained by stimulating 10 times at $120 \%$ of the resting threshold, was the motor evoked potential. If the resting threshold could be obtained from the affected part, it became the positive group; and if there was no response to a maximum magnetic stimulus, and the resting threshold could not be obtained, it became the negative group. Also, the FIM, activities of daily living (ADL) evaluation tool, was performed for all patients when the motor evoked potential test was conducted. The FIM scale uses 7 scales to evaluate the 18 items, assessing whether the patient needs help or aid to perform daily activities, on a scale from 7 to 126 points [11]. The 18 components of FIM include self-care, sphincter control, mobility-transfer, locomotion, communication, and social cognition [11].

The evaluation was conducted by the same tester through the interviews. The FIM evaluation repeated after a month of comprehensive rehabilitation. Among the stroke patients who had undergone the transcranial magnetic stimulus, those for whom the resting threshold could be obtained according to the presence or absence of the motor evoked potential were classified as the motor evoked potential positive group (MEP positive group, 
P-MEP). Those for whom the resting threshold could not be obtained with the maximum magnetic stimulus (i.e., $100 \%$ stimulus) were classified as the motor evoked potential negative group (N-MEP).

The changes in the FIM score for the one month-long comprehensive rehabilitation were obtained, and compared to the motor evoked potential measurements. Depending on the presence or absence of the motor evoked potential, the FIM and subitems were compared using the Wilcoxon signed rank test, and the changes in FIM and subitems were compared using the Mann-Whitney test. SPSS ver. 18.0 (SPSS Inc., Chicago, IL, USA) was used for statistical analysis, and there was statistical significance when $\mathrm{p}<0.05$.

\section{RESULTS}

A total of 9 patients were in the P-MEP group, which had the motor evoked potential induced by the transcranial magnetic stimulus; and a total of 10 patients were in the N-MEP group, which had no motor evoked potential induced by the maximum transcranial magnetic stimulus. The mean resting threshold obtained from the cerebral cortex damaged by the stroke was $71.7 \%$ of the maximum stimulus. The mean age of the P-MEP group with the motor evoked potential induced was 56.3 years, and the mean age of the N-MEP group was 56.2 years. No significant difference between the two groups was observed. The motoricity index (MI), measured to evaluate the motor skills at transfer, were 56.4 points in the P-MEP group and 40.9 points in the N-MEP group. The difference was statistically significant (Table 1). The initial and final FIM

Table 1. Demographic and clinical characteristics of subjects

\begin{tabular}{lcc}
\hline \multicolumn{1}{c}{ Characteristic } & $\begin{array}{c}\text { N-MEP } \\
(\mathbf{n = 1 0 )}\end{array}$ & $\begin{array}{c}\text { P-MEP } \\
(\mathbf{n}=\mathbf{9})\end{array}$ \\
\hline Gender (M:F) & $5: 5$ & $5: 4$ \\
Age (yr) & $56.2 \pm 17.8$ & $56.3 \pm 14.0$ \\
Type of stroke & - & - \\
Hemorrhage & 2 & 4 \\
Infarction & 8 & 5 \\
Resting motor threshold (\%) & - & $71.7 \pm 18.4$ \\
Duration from onset (day) & $34.3 \pm 26.2$ & $28.6 \pm 13.5$ \\
\hline Motoricity index & $40.9 \pm 15.7$ & $56.4 \pm 16.7^{*}$ \\
\hline
\end{tabular}

Values are presented as number or mean \pm standard deviation.

MEP, motor evoked potential; N-MEP, MEP-negative group; P-MEP, MEP-positive group.

${ }^{*} \mathrm{p}<0.05$ from the Mann-Whitney test.

Table 2. Comparison of FIM and subitem score in P-MEP and N-MEP group

\begin{tabular}{|c|c|c|c|c|c|}
\hline \multirow{2}{*}{ FIM score } & & \multicolumn{2}{|c|}{ N-MEP } & \multicolumn{2}{|c|}{ P-MEP } \\
\hline & & Mean \pm SD & p-value & Mean \pm SD & p-value \\
\hline \multirow[t]{2}{*}{ Total FIM } & Initial & $61.2 \pm 18.9$ & $0.005^{*}$ & $59.6 \pm 10.1$ & $0.008^{*}$ \\
\hline & Final & $74.5 \pm 20.3$ & & $92.2 \pm 20.1$ & \\
\hline \multirow[t]{2}{*}{ Self-care } & Initial & $15.3 \pm 4.9$ & $0.005^{*}$ & $14.6 \pm 3.8$ & $0.008^{*}$ \\
\hline & Final & $21.6 \pm 5.7$ & & $25.8 \pm 8.4$ & \\
\hline \multirow[t]{2}{*}{ Sphincter control } & Initial & $9.8 \pm 5.2$ & 0.854 & $9.6 \pm 3.6$ & $0.027^{*}$ \\
\hline & Final & $10.0 \pm 4.3$ & & $13.0 \pm 1.7$ & \\
\hline \multirow[t]{2}{*}{ Mobility-transfer } & Initial & $8.5 \pm 3.8$ & $0.015^{*}$ & $7.4 \pm 3.0$ & $0.011^{*}$ \\
\hline & Final & $11.5 \pm 4.2$ & & $13.6 \pm 4.9$ & \\
\hline \multirow[t]{2}{*}{ Locomotion } & Initial & $3.3 \pm 1.8$ & $0.027^{*}$ & $3.4 \pm 1.6$ & $0.018^{*}$ \\
\hline & Final & $4.6 \pm 2.9$ & & $7.3 \pm 3.9$ & \\
\hline \multirow[t]{2}{*}{ Communication } & Initial & $9.8 \pm 3.3$ & 0.066 & $9.8 \pm 2.9$ & $0.026^{*}$ \\
\hline & Final & $10.9 \pm 3.0$ & & $12.2 \pm 1.6$ & \\
\hline \multirow[t]{2}{*}{ Social cognition } & Initial & $14.5 \pm 5.2$ & 0.066 & $14.0 \pm 3.7$ & $0.016^{*}$ \\
\hline & Final & $16.2 \pm 5.5$ & & $18.4 \pm 2.3$ & \\
\hline
\end{tabular}

SD, standard deviation; FIM, Functional Independence Measure; MEP, motor evoked potential; N-MEP, MEP-negative group; P-MEP, MEP-positive group.

${ }^{*} \mathrm{p}<0.05$ from the Wilcoxon signed rank test. 


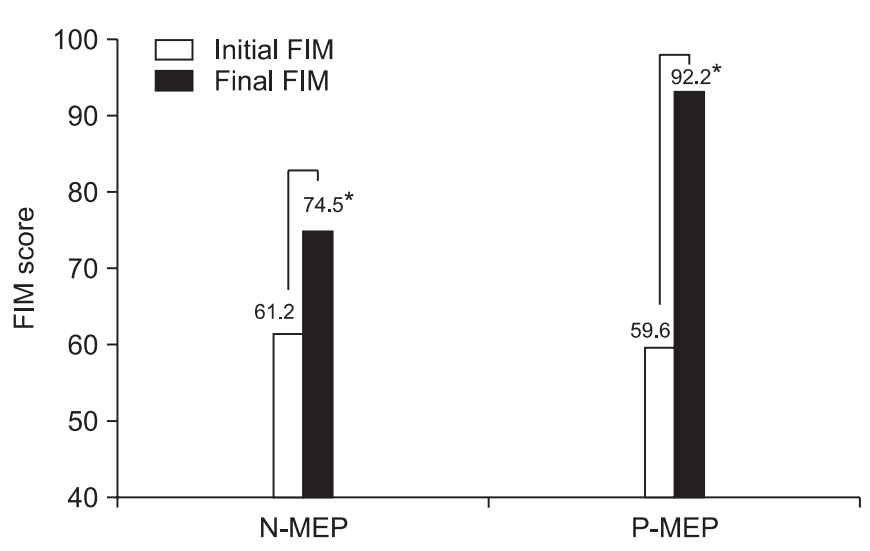

Fig. 1. There is significant improvement of FIM score in P-MEP and N-MEP group. FIM, Functional Independence Measure; MEP, motor evoked potential; N-MEP, MEP-negative group; P-MEP, MEP-positive group. * $\mathrm{p}$ value $<0.05$.

Table 3. Comparison of FIM change between the two groups

\begin{tabular}{lrcc}
\hline & N-MEP & P-MEP & p-value \\
\hline FIM score change & $13.3 \pm 6.3$ & $32.7 \pm 15.2$ & $0.013^{*}$ \\
Self-care change & $6.3 \pm 2.9$ & $11.2 \pm 5.8$ & $0.028^{*}$ \\
Sphincter control change & $0.2 \pm 2.6$ & $3.4 \pm 3.2$ & 0.065 \\
Mobility-transfer change & $3.0 \pm 2.4$ & $6.1 \pm 4.8$ & 0.182 \\
Locomotion change & $1.3 \pm 1.4$ & $3.9 \pm 3.8$ & 0.211 \\
Communication change & $1.1 \pm 1.9$ & $2.4 \pm 2.6$ & 0.211 \\
Social cognition change & $1.7 \pm 2.9$ & $5.2 \pm 4.2$ & 0.65 \\
\hline
\end{tabular}

Values are presented as mean \pm standard deviation.

FIM, Functional Independence Measure; MEP, motor evoked potential; N-MEP, MEP-negative group; P-MEP, MEP-positive group.

*p $<0.05$ from the Mann-Whitney test.

scores of the P-MEP group with the motor evoked potential induced were 59.6 and 92.2, respectively; and the final FIM score was significantly higher than the initial FIM score. The initial and final FIM scores of the N-MEP group with no motor evoked potential induced were 61.2 and 74.5, respectively; and the final value was significantly higher than the initial value (Table 2, Fig. 1). The change in the two groups, obtained by subtracting the initial FIM score and the later score, was $32.7 \pm 15.2$ in the P-MEP group, and $13.3 \pm 6.3$ in the N-MEP group. The PMEP group value was significantly higher than that of $\mathrm{N}$ MEP (Table 3). When the change in the two groups, obtained by subtracting the initial values and later values of the detailed FIM items, were compared, the P-MEP group

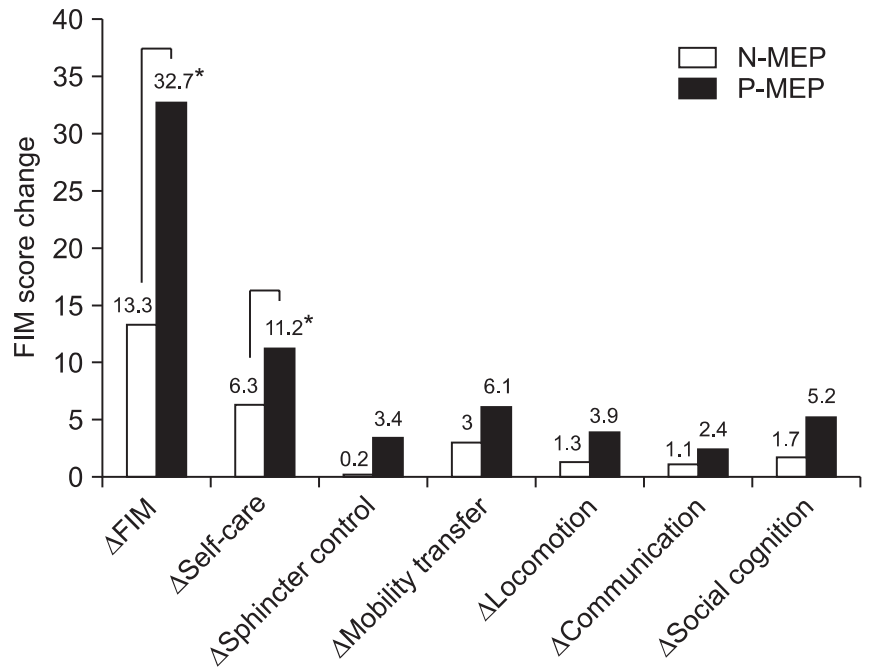

Fig. 2. FIM score and self-care score change is improved in P-MEP group compaired to N-MEP group. FIM, Functional Independence Measure; MEP, motor evoked potential; N-MEP, MEP-negative group; P-MEP, MEPpositive group. *p-value $<0.05$.

value was significantly higher than the $\mathrm{N}$-MEP value in the self-care (Table 3, Fig. 2).

When the initial and final values of FIM subitems were compared in the P-MEP group, the final values were significantly higher than the initial values in all areas, i.e., self-care, sphincter control, mobility-transfer, locomotion, communication, and social cognition (Table 2). In the N-MEP group, the final values were significantly higher than the initial values only in terms of self-care, mobility-transfer, and locomotion (Table 2).

\section{DISCUSSION}

This study compared the motor evoked potential of stoke patients and the level of recovery of the physical and cognitive functions necessary for conducting daily activities, and evaluated their clinical values, and attempted to help establish rehabilitation plans for the stroke patients. With regard to the stroke patients in the subacute period, the ADL of the group with the motor evoked potential measured in the cerebral motor neurons was better than that of the group with no motor evoked potential in the cerebral motor neurons. The change was significant in self-care, and the improvement of the social cognitive function and communication was not significantly related to the presence or absence of the motor evoked potential. 
In this study, in both the group with and without the motor evoked potential of the affected abductor pollicis brevis induced by the parietal region magnetic stimulus, the final FIM value was significantly higher than the initial FIM value. Among the patients in the subacute period, the 4-week rehabilitation was effective in both groups. Also, the change of the initial and final value of ADL was significantly higher in the P-MEP group than in the N-MEP group. It is consistent with other studies [12] that reported that the motor evoked potential measured in the early phases of the disease can be used as a factor predicting the stroke patient's recovery of the upper limb motor skills $[13,14]$. The MBI score was higher at discharge in the P-MEP group than in the N-MEP group in the early phases of the disease. Also, the P-MEP group was thought to have improved more than the N-MEP group, when they received the same rehabilitation treatment.

According to this study, when the initial and final values of detailed FIM items were compared in the P-MEP group, there was a significant improvement in all areas, i.e., self-care, sphincter control, mobility-transfer, locomotion, communication, and social cognition. In the $\mathrm{N}$-MEP group, there was a significant improvement only in self-care, mobility-transfer, and locomotion. In the P-MEP group, the stroke patients in the subacute period could be viewed as responding well to the comprehensive rehabilitation treatment in the cognitive and language functions, as well as the motor skills.

As for changes in the detailed FIM items, when the P-MEP group was compared to the N-MEP group, there was a significant improvement only in self-care. The motor evoked potential is the level of activity of the overall motor nervous system connected to the cerebral cortex, brain, and spinal cord, and the motor evoked potential was not induced perhaps because the nerve cells or nerve trunks are dead, or have a very high motor threshold $[6,15]$. Accordingly, the motor nervous system of the P-MEP group was thought to be more active than that of the N-MEP group. This study showed that there was more improvement in self-care (which corresponds to the motor FIM of the FIM subitems); and the changes in the communication and social cognition (which correspond to the cognitive FIM) was not affected by the presence or absence of the motor evoked potential.

Previous studies on the motor evoked potential and prognosis [16] argued that the presence or absence of motor evoked potential response was an important factor in the early phases of the diseases in predicting the prognosis of the stroke patients. Kang et al. [5] reported that, when the degree of functional recovery was compared per the presence or absence of the motor evoked potential, the degree of functional recovery, as measured by MBI two months later, was better in the P-MEP group.

Jung et al. [15] held that, if there was motor evoked potential, the National Institute of Health Stroke Scale (NIHSS) test and the MEP threshold were correlated, and that the NIHSS showed the prognostics of the stroke patient. Lee and Jung [7] and Lee et al. [8] argued that, depending on the presence or absence of the somatosensory evoked potential and motor evoked potential measured in the hemiplegic upper limb, there was a significant difference between the initial Manual Function Test (MFT) and the final MFT, and that it is more beneficial to conduct the two tests at the same time, and evaluate the test results comprehensively. In this study, the FIM scores of the patients with the motor evoked potential improved more, and the self-care function (part of the motor skills) improved. This result is consistent with the previous studies related to the motor evoked potential and functional prognosis.

The significance of this study lies in the fact that the motor evoked potential has been used for the first time to investigate the prognostics of the motor skills and cognitive function among the ADL functions. It also researched the prognosis of motor skills and cognitive functions of patients in the subacute period. It may be possible to use an objective measurement tool closely related to the cortical excitability, such as the motor evoked potential, to predict the prognosis of the stroke patients. This can make up for the limitations of the tools measuring clinical movement, and the radiological tools in prognosis and prediction. Even though the initial motor skills of patients in the subacute period are not good, if motor evoked potential is induced, it can be expected that ADL will improve and rehabilitation treatment will be effective. This study made it possible to compare detailed FIM items to see their effects on other items. The patients improved in self-care related to motor skills, but the items related to the cognitive function were not greatly affected.

The limitation of this study is that the number of patient groups was too small, and that functional recovery depending on the location of the stroke was not studied. In this study, the subjects were patients who had infarction 
of the middle cerebral artery and cerebral hemorrhage in the basal ganglia. The P-MEP group had less infarction and more cerebral hemorrhage than the N-MEP. As prognosis may vary depending on the location of the brain lesion it may affect the results, and the size of the brain lesion may also affect prognosis. This was not further investigated, presenting a limitation of this study. Also, the FIM score, an indicator of motor skills measured soon after referral, was not significantly different between the two groups, but the MI was higher in the P-MEP group, which possibly affected the results. Additional research on the functional recovery and cognitive evoked potential as depending on the location and size of the stroke is necessary.

In conclusion, this study analyzed the correlation between motor evoked potential and ADL in stroke patients, and found that among stroke patients in the subacute period, ADL scores improved more in the P-MEP group than in the N-MEP group. Accordingly, early measurement of MEP will be instrumental in predicting the prognosis of stroke patients in the subacute period.

\section{CONFLICT OF INTEREST}

No potential conflict of interest relevant to this article was reported.

\section{REFERENCES}

1. Diamond PT, Felsenthal G, Macciocchi SN, Butler DH, Lally-Cassady D. Effect of cognitive impairment on rehabilitation outcome. Am J Phys Med Rehabil 1996;75:40-3.

2. Stinear C. Prediction of recovery of motor function after stroke. Lancet Neurol 2010;9:1228-32.

3. Chu NS. Motor evoked potentials with magnetic stimulation: correlations with height. Electroencephalogr Clin Neurophysiol 1989;74:481-5.

4. Curra A, Modugno N, Inghilleri M, Manfredi M, Hallett M, Berardelli A. Transcranial magnetic stimulation techniques in clinical investigation. Neurology 2002;59:1851-9.

5. Kang MJ, Yoon TS, Park CI, Shun SI. Motor evoked potential in stroke. J Korean Acad Rehabil Med 1993;17:26-35.

6. Han TR, Bang MS, Lee KW. Motor evoked potentials of upper and lower extremities by magnetic stimulation in hemiparesis. J Korean Acad Rehabil Med 1998;22:386-91.

7. Lee JJ, Jung HY. The correlation of hemiplegic upper limb recovery with SEP and MEP in subjects with a stroke. J Korean Acad Rehabil Med 2008;32:512-7.

8. Lee SY, Lim JY, Kang EK, Han MK, Bae HJ, Paik NJ. Prediction of good functional recovery after stroke based on combined motor and somatosensory evoked potential findings. J Rehabil Med 2010;42:16-20.

9. Hsieh YW, Wu CY, Lin KC, Chang YF, Chen CL, Liu JS. Responsiveness and validity of three outcome measures of motor function after stroke rehabilitation. Stroke 2009;40:1386-91.

10. Granger CV, Cotter AC, Hamilton BB, Fiedler RC. Functional assessment scales: a study of persons after stroke. Arch Phys Med Rehabil 1993;74:133-8.

11. Hamilton BB, Granger CV. Disability outcomes following inpatient rehabilitation for stroke. Phys Ther 1994;74:494-503.

12. Kim C, Jeong J. The significance of motor evoked potentials as a prognostic factor in the early stage of stroke patients. J Korean Acad Rehabil Med 1999;23:1213-20.

13. Brouwer BJ, Schryburt-Brown K. Hand function and motor cortical output poststroke: are they related? Arch Phys Med Rehabil 2006;87:627-34.

14. Vang C, Dunbabin D, Kilpatrick D. Correlation between functional and electrophysiological recovery in acute ischemic stroke. Stroke 1999;30:2126-30.

15. Jung HY, Kim TH, Park JH. Relationship of National Institute of Health Stroke Scale and motor evoked potentials in subjects with stroke. J Korean Acad Rehabil Med 2005;29:563-7.

16. Kandler RH, Jarratt JA, Davies-Jones GA, Gumpert EJ, Venables GS, Sagar HJ, et al. The role of magnetic stimulation as a quantifier of motor disability in patients with multiple sclerosis. J Neurol Sci 1991;106:314. 